\title{
Genesis
}

Manuscrits - Recherche - Invention

\section{Pleins et déliés dans les manuscrits de D’Alembert}

\section{Olivier Ferret, Alexandre Guilbaud et Irène Passeron}

\section{(2) OpenEdition}

\section{Journals}

\section{Édition électronique}

URL : http://journals.openedition.org/genesis/922

DOI : 10.4000/genesis.922

ISSN : 2268-1590

\section{Éditeur :}

Presses universitaires de Paris Sorbonne (PUPS), Société internationale de génétique artistique littéraire et scientifique (SIGALES)

\section{Édition imprimée}

Date de publication : 10 avril 2012

Pagination : 67-82

ISBN : 978-2-84050-822-9

ISSN : 1167-5101

\section{Référence électronique}

Olivier Ferret, Alexandre Guilbaud et Irène Passeron, «Pleins et déliés dans les manuscrits de D'Alembert », Genesis [En ligne], 34 | 2012, mis en ligne le 10 avril 2014, consulté le 12 juin 2020. URL http://journals.openedition.org/genesis/922 ; DOl : https://doi.org/10.4000/genesis.922 


\title{
Pleins et déliés dans les manuscrits de D'Alembert*
}

\author{
Olivier Ferret, Alexandre Guilbaud, Irène Passeron
}

$\mathrm{D}$ 'Alembert ${ }^{1}$ a laissé une petite dizaine de milliers de folios manuscrits, dont l'analyse soulève des questions relatives à l'histoire de leur conservation mais aussi à la définition même de ce que l'on appelle un « manuscrit de D'Alembert » : ceux qui résultent de ses activités en tant que membre de l'Académie royale des sciences de Paris, de l'Académie des sciences et belleslettres de Berlin et de l'Académie française ; ceux qu'il a envoyés à des destinataires privés; ceux enfin qu'il a conservés chez lui et qui ont été transmis à Condorcet (1743-1794), son légataire universel.

Ces derniers sont pour la plus grande partie entrés à la Bibliothèque de l'Institut de France (Institut) avec ceux de Condorcet, ce qui explique qu'ils s'y trouvent parfois mélangés 2 . Une autre partie a intégré la Bibliothèque nationale de France (BnF), d'autres bibliothèques publiques ou des collections privées. Outre les lettres de D'Alembert envoyées dans toute l'Europe, la plupart des autres manuscrits connus sont conservés aux archives de l'Académie française et de l'Académie des sciences de Paris, et à la Berlin-Brandenburgische Akademie der Wissenschaften (BBAW).

À l'Institut, la plupart des papiers dits « de D'Alembert » sont reliés sous les cotes Ms. 1786 à 1794 (trois mille folios environ) et Ms. 2466 à 2473 (deux mille trois cents folios environ). Les regroupements, effectués après la mort de D'Alembert, parfois par « genre », scientifique ou littéraire, parfois par ordre alphabétique (de correspondants pour les lettres du Ms. 2466), ne facilitent pas la datation de leur rédaction. Il est cependant certain, soit par l'analyse du contenu, soit par les dates portées sur les lettres, que l'essentiel de ce fonds est postérieur à 1770 .

Se pose aussi, d'un point de vue théorique, la question de ce qu'il faut entendre par «manuscrit de D'Alembert». À ce niveau, deux interprétations au moins peuvent entrer en conflit, celle qui donne le primat à l'auctorialité et celle qui se fonde sur la matérialité de l'archive. Selon la première, le corpus est défini à partir de la notion d'auteur et ne retient que des textes où prime l'idée de création : que l'auteur s'exprime à travers ses notes de blanchisserie ou à travers un récit fictionnel original est un débat interne à cette interprétation. La valeur intellectuelle du texte est d'abord jaugée à l'aune de l'originalité, ce qui sous-entend une définition forte de l'auctorialité. Lorsqu'un manuscrit n'est pas de la main de l'auteur, sauf s'il est dicté, il est ravalé au rang de simple « copie » d'un « original », et peut même se trouver rejeté du corpus si cet original a par ailleurs été conservé. Suivant la même logique, si le scripteur n'est pas à l'origine du texte, s'il copie, voire traduit, modifie ou commente le texte d'un autre, le manuscrit perd également de sa valeur.

Selon la seconde interprétation, le corpus est défini plus largement comme une production écrite, ce qui, dans le cas de D'Alembert, est justifié par une activité liée à deux espaces de travail et de réflexion, celui des académies et celui d'échanges divers : les discussions autour de lettres et de textes copiés, en particulier dans les salons ; les correspondances entre personnes privées ou avec les périodiques.

\footnotetext{
* Nous tenons à remercier Michelle Chapront-Touzé, Annie Chassagne, Pierre Crépel, Claudette Fortuny, Christian Gilain, Marie Leca-Tsiomis, Nicolas Rieucau, Jean-Pierre Schandeler et Catherine Volpilhac-Auger pour leurs relectures attentives de cet article. Nous remercions aussi François Prin pour ses clichés des six illustrations qu'il comporte.

1. Pour plus d'informations sur D'Alembert (1717-1783), sa biographie, sa bibliographie et ses œuvres en cours de publication (Paris, CNRS Éditions), nous renvoyons au site <http://dalembert.obspm.fr/>. Les Euvres complètes de D'Alembert (O.C.) comportent cinq séries : traités et mémoires mathématiques, 1736-1756 (I), articles de l'Encyclopédie (II), Opuscules et mémoires mathématiques, 1757-1783 (III), écrits philosophiques, historiques et littéraires (IV), correspondance générale (V). 2. Voir l'histoire mouvementée du fonds dans O.C., vol. V/1, Inventaire analytique de la correspondance, 1741-1783, dir. I. Passeron, A.-M. Chouillet, J.-D. Candaux, 2009, Introduction générale, p. XX-XXIII.
} 
L'enquête que nous proposons tentera de s'affranchir de cette distinction et s'efforcera d'articuler trois ordres de considérations ${ }^{3}$. Les unes sont relatives à la matérialité des documents : outre les problèmes liés à l'histoire, rappelée plus haut, de la conservation des « papiers de D'Alembert », elles soulèvent la question de la présentation matérielle des manuscrits et des enseignements que l'on peut en tirer. D'autres concernent l'interprétation des divers phénomènes textuels que révèle l'étude des remaniements dont les manuscrits portent la trace : elles conduisent à émettre des hypothèses relatives aux stratégies d'écriture mises en œuvre par D'Alembert. L'examen du passage du manuscrit à l'imprimé, ou, à l'inverse, la mise au jour de textes demeurés inédits du vivant de l'auteur amènent enfin à s'interroger sur les choix de publication effectués par D'Alembert, que l'on peut tenter de formuler en termes de stratégies éditoriales. Sans viser à l'exhaustivité, tout en cherchant à présenter des exemples illustrant la variété des fonds conservés, nous envisagerons successivement trois ensembles textuels : le corpus scientifique, le corpus encyclopédique et le corpus des éloges académiques.

\section{Le corpus scientifique}

Les manuscrits « scientifiques 4 » de D'Alembert, qu'il s'agisse de manuscrits parvenus jusqu'à nous ou de manuscrits dont nous n'avons pu retrouver que des traces indirectes, couvrent l'ensemble de sa carrière, de ses premiers écrits de jeunesse, non publiés, jusqu'à la version autographe, non achevée, d'un neuvième tome d'Opuscules mathématiques (Opuscules). Entre ces deux bornes, sa production scientifique connaît plusieurs périodes bien identifiées 5 : celle de ses grands traités et mémoires académiques, qui s'achève avec la publication du troisième tome des Recherches sur le système du monde en 1756 ; la phase de transition des années 1750 , marquée par la dégradation de ses relations avec l'Académie des sciences de Paris et l'Académie de Berlin ainsi que par son investissement dans l'entreprise encyclopédique ; la période, enfin, de ses Opuscules, recueils de mémoires sur différents sujets dont il fait paraître huit tomes entre 1761 et 1780 et qui marquent un changement notable de mode de publication, consécutif à sa prise de distance avec les deux académies parisienne et berlinoise.
Nous nous attacherons ici à trois ensembles représentatifs de cette temporalité : manuscrits de jeunesse, manuscrits de la période 1749-1757 et manuscrits tardifs. Les exemples particulièrement révélateurs que nous avons choisi de présenter dans ce cadre nous permettront non seulement de soulever la question des liens que les manuscrits de D'Alembert entretiennent avec ses modes d'activité scientifique, mais aussi de montrer comment ces mêmes matériaux nous renseignent sur l'évolution de ses stratégies d'écriture.

À l'exception de deux textes autographes, «Remarques et éclaircissemens sur differens endroits de l'application de l'algèbre à la géométrie de M. Guisnée » (daté de 1736), et «Remarques sur quelques endroits des Principes de Newton » (postérieur à 1739)6, les premiers manuscrits scientifiques de D'Alembert sont aujourd'hui perdus. C'est le cas de trois mémoires sur le calcul intégral, rédigés avant son entrée à l'Académie des sciences de Paris (en mai 1741, comme adjoint astronome), mais dont nous pouvons néanmoins nous faire une première idée grâce aux rapports qui ont été copiés dans les Registres [manuscrits] de l'Académie royale des sciences de Paris (Registres). Cinq autres mémoires concernent le problème de la réfraction des corps solides (c'est-à-dire leur mouvement dans des milieux de différentes densités). Deux sont introuvables bien que leur existence soit attestée par des rapports académiques de 1740. Les trois suivants, lus à l'Académie en 1740,1741 et 1742 , sont intégralement transcrits dans les Registres.

Ces différents matériaux, pour la plupart indirects et lacunaires, fournissent des informations précieuses sur les premières lectures et les premiers sujets de recherche de

3. Voir aussi, sur un autre corpus, N. Rieucau, « La neige avait-elle recouvert le volcan? L'écriture par Condorcet du Tableau historique des progrès de l'esprit humain », Genesis, $\mathrm{n}^{\circ} 22$, « Philosophie », 2003, p. 13-35.

4. D'après la structuration des Euvres complètes (voir, ci-dessus, n. 1), seront ici considérés comme tels les manuscrits relatifs aux textes publiés dans les séries I et III, à l'exception de l'œuvre encyclopédique, de la correspondance, des écrits philosophiques, historiques et littéraires ainsi que de la musique.

5. Voir O.C., vol. III/I, Opuscules mathématiques, t. I (1761), dir. P. Crépel,

A. Guilbaud, Guillaume Jouve, 2008, p. XV-XXXIII.

6. Institut, Ms. 2033 et Ms. 2467. 
D’Alembert. Ils feront par ailleurs, à des degrés divers, l'objet de réemplois ultérieurs. Nous savons d'une part que D'Alembert confiera à Bougainville plusieurs de ses mémoires non imprimés afin qu'il les utilise pour la rédaction d'un Traité du calcul intégral que D'Alembert appelle lui-même de ses vœux dans l'Encyclopédie (1751) et qui ne paraîtra, en deux tomes, qu'en 1754 et 1756 . Il semble que ses trois premiers écrits sur le sujet en aient fait partie. Des passages des trois mémoires sur la réfraction des corps copiés dans les Registres se retrouveront d'autre part dans le Traité des fluides de D'Alembert (1744)7, l'un de ses six principaux traités de la première période de son activité scientifique.

Les manuscrits de la période 1749-1757 mettent en perspective la dégradation des relations de D'Alembert avec Euler et l'Académie de Berlin, dont ce dernier dirige alors la classe de mathématiques. À l'origine, le report du prix de Berlin de 1750, portant sur la résistance des fluides : D'Alembert y avait soumis une pièce en latin intitulée « Theoria resistentiae », datée du 25 novembre 1749 et actuellement conservée à la BBAW (cote I-M478). Mécontent de la décision et furieux contre Euler, l'un des trois commissaires du concours, D'Alembert retire sa pièce en 1751 , l'enrichit significativement avant de la faire paraître l'année suivante, en langue française, sous le titre d'Essai d'une nouvelle théorie de la résistance des fluides (1752). Dans le chapitre IX, l'une des deux additions les plus importantes de l'ouvrage, il affirme « qu'il lui est tombé entre les mains [...] une théorie » sur le mouvement des rivières s'inspirant largement de la méthode exposée dans sa pièce de 1749, théorie manuscrite perdue qui n'est autre qu'un mémoire d'Euler daté d'août 1750, publié seulement en 1767. L'enjeu est ici une question de priorité : celle, revendiquée par D'Alembert, de l'établissement des premières équations aux dérivées partielles gouvernant le mouvement des fluides. La pièce de 1749 démontre l'influence décisive des recherches de D'Alembert sur celles d'Euler8, qui les généralisera ensuite dans trois célèbres mémoires 9 .

Bien que l'épisode du prix de 1750 provoque la brouille des deux savants pendant une douzaine d'années, des contacts indirects et tumultueux se poursuivent, ce qu'illustre en particulier le manuscrit autographe du mémoire «Sur les logarithmes des quantités négatives », daté du 16 juin 1752, envoyé à Berlin et conservé à la BBAW (cote I-M5). D'Alembert y poursuit un débat scientifique avec Euler, entamé dans leur correspondance des années 1747 et 1748, mais auquel ce dernier ne répondra pas. Non publié, le manuscrit sera néanmoins largement réutilisé pour le mémoire que le savant consacre à ce sujet dans le premier tome de ses Opuscules $(1761)^{10}$.

Parmi les autres manuscrits en notre possession pour la période 1749-1757, deux concernent enfin la polémique opposant D'Alembert, Euler et Daniel Bernoulli sur le problème des cordes vibrantes. Dans sa lettre à Formey, secrétaire de l'Académie de Berlin, du 6 novembre 1755, l'encyclopédiste fait parvenir un mémoire manuscrit portant de nombreuses corrections, daté de deux jours plus tôt, ayant pour titre «Observations sur deux mémoires de MM. Euler et D. Bernoulli, insérés dans les Mémoires de 1753 », et réfutant les critiques formulées par les deux savants à l'encontre de sa théorie - elle-même publiée dans les volumes de l'Académie de Berlin pour les années 1747 et 1750 . Bien que les lettres de Formey n'aient pas été retrouvées, celles que lui adresse D'Alembert au cours des deux années suivantes nous apprennent qu'il consent le 26 novembre 1756 à ce que ses « Observations » ne soient pas imprimées à condition qu'elles lui soient retournées avec la réponse d'Euler, puis qu'il renvoie le mémoire légèrement modifié le 5 janvier 1757. Un mois plus tard, il renonce finalement à la publication et obtient l'impression d'une courte lettre qui marquera sa rupture définitive avec l'Académie de Berlin. Par chance, deux manuscrits de la BBAW (cotes I-M6 et I-M7) correspondent respectivement à la copie du texte de D'Alembert du 4 novembre 1755 et à la version renvoyée à Berlin en 1757. Leur examen comparé

7. Voir le volume I/4a des O.C. (Textes de mathématiques pures 1745 1752, 2007), édité par Ch. Gilain, pour ce qui concerne les mémoires sur le calcul intégral.

8. Voir G. Grimberg, «D’Alembert et les équations aux dérivées partielles en hydrodynamique », thèse de doctorat, Université Paris VII, 1998.

9. Euler, «Principes généraux de l'état d'équilibre des fluides », "Principes généraux du mouvement des fluides » et « Continuations des recherches sur la théorie du mouvement des fluides », Histoire de l'Académie des sciences et belles-lettres de Berlin pour l'année 1755 (1757), p. 274-361.

10. Voir $O . C$., vol. III/1, Mémoire 6 : « Sur les Logarithmes des quantités négatives ». 
permet non seulement d'identifier la seconde comme le manuscrit autographe de 1755 retourné à Paris, retouché puis renvoyé par D'Alembert, mais aussi de distinguer les corrections originales du savant : des corrections mineures, mais significatives, apportées fin 1756 début 1757. Ils formeront en outre la version préliminaire des « Recherches sur les vibrations des cordes sonores », publiées dans le premier tome de ses Opuscules ${ }^{11}$.

Ces différents manuscrits permettent donc de suivre, au cours des décennies 1740 et 1750 , la polarisation de l'activité scientifique de D'Alembert autour des académies de Paris et Berlin, et d'un homme, Euler, puis la dégradation de leurs rapports à partir de l'épisode du prix sur la résistance des fluides. Mieux encore, ils permettent de mettre en évidence le réemploi quasi systématique, dans des écrits ultérieurs, de travaux qu'il n'a pas souhaité ou pas pu faire publier. Ils permettent encore d'émettre des hypothèses sur la teneur des manuscrits perdus, d'étudier l'évolution des idées de l'auteur d'une version à l'autre et de mieux comprendre les querelles de priorité dont D'Alembert se montre coutumier. Cette stratégie d'écriture est à rapprocher de celle mise en œuvre dans la fabrication d'autres productions académiques, celle de certains de ses Éloges, comme nous le verrons plus loin.

Ces manuscrits scientifiques soulèvent aussi le problème, déjà évoqué, de ce qui peut ou non être considéré comme un manuscrit de D'Alembert. Les nombreuses copies dans les Registres de manuscrits non retrouvés ne bénéficient certes pas du même statut que les manuscrits originaux absents : elles n'en constituent que des retranscriptions plus ou moins fidèles. Que dire en revanche des quelque cent soixante rapports auxquels D'Alembert participe entre 1741 et 1783 en tant que membre des commissions d'expertise (de mémoires, d'ouvrages, de machines et de prix) de l'Académie des sciences de Paris ? S'ils sont presque tous copiés dans les Registres, la moitié d'entre eux environ nous est également parvenue sous forme manuscrite. Certains sont autographes, certains de la main d'un autre commissaire, mais signés par D'Alembert, quelques autres, plus rares, de la main d'un autre commissaire et non signés par le savant. Nous disposons ainsi d'un nouvel ensemble de manuscrits scientifiques directement liés à son activité d'académicien et de savant.
Outre ces rapports et plusieurs lettres de sa correspondance, les manuscrits scientifiques les plus tardifs sont pour la plupart conservés dans les «papiers D'Alembert » de l'Institut, sous les cotes Ms. 1787 à 1793. Ils contiennent notamment (Ms. 1788 et 1789) les neuf cents feuillets d'un manuscrit autographe partiel du huitième tome des Opuscules, dont les ajouts et corrections forment, après reconstitution, un texte identique aux passages correspondants de la version imprimée (la moitié $\mathrm{du}$ volume environ). Le manuscrit porte des mentions de pagination et de signatures ${ }^{12}$ coïncidant parfaitement avec celles du texte publié (fig. 1), mentions dont on peut penser qu'elles ont été ajoutées sur un manuscrit confié à l'imprimeur et rendu ensuite au savant. Les derniers feuillets renferment les planches du volume, sous la forme d'un assemblage de figures manuscrites découpées, collées et agencées par D'Alembert, avec des notes de régie autographes (Ms. 1788, f. 519 : fig. 2).

La partie la plus conséquente de ces papiers tardifs (Ms. 1790 à 1793) correspond néanmoins à un neuvième tome d'Opuscules resté inédit, rassemblant les manuscrits autographes de quarante paragraphes de longueurs très variables, « revu[s] » une ou deux fois pour la plupart, et caractéristiques du style commun aux huit autres tomes : des remarques sur différents sujets, souvent en lien avec une polémique ou une question de priorité, des éclaircissements sur un passage des tomes précédents, nécessitant en général d'avoir celui-ci « sous les yeux », ou encore de simples réflexions écrites au fil de la plume, sans travail de composition et sans souci d'intelligibilité. D'Alembert n'écrit, semble-t-il, que pour quelques « géomètres » dont Euler et Lagrange, son correspondant scientifique le plus assidu depuis le début des années 1760. Il rédige pour publier rapidement : nous sommes loin ici des stratégies d'écriture et de publication sur le long terme mises en œuvre dans les années 1740 et 1750 .

Ce neuvième tome inédit nous renseigne sur ses derniers travaux où se retrouvent ses sujets de prédilection, tels le problème des cordes vibrantes et de la propagation du son, les principes de la mécanique, la mécanique

11. Voir O.C., vol. III/1, Mémoire 1.

12. Marques portées au recto des premiers feuillets de chaque cahier imprimé, du type « $\mathrm{a} », \ll \mathrm{b} »$, etc., « aa », etc. 


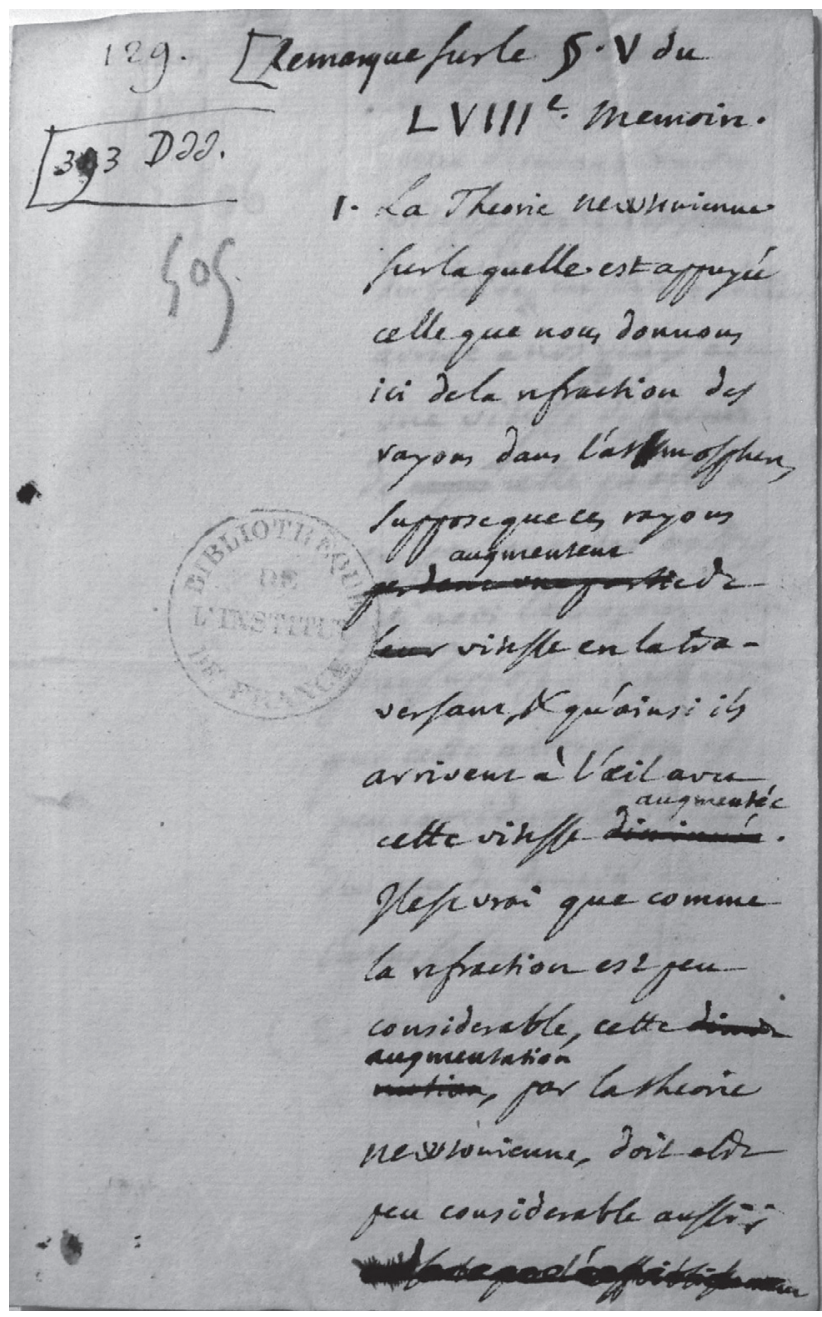

Fig. 1 : D'Alembert, Opuscules, t. VIII, Appendice, manuscrit autographe du début de la « Remarque sur le $\S$. V du LVIIIe Memoire»; le folio porte une indication de changement de cahier conforme à l'imprimé (Institut de France, ms. 1788, f. 505)

céleste, l'hydrodynamique, l'optique, le calcul différentiel et intégral et les probabilités. Le dernier des quarante paragraphes contient en outre une sorte de « testament scientifique » revendiquant ses propres apports en hydrodynamique, signe qu'au crépuscule de sa vie, l'épisode du prix de l'Académie de Berlin de 1750 n'a pas fini de le préoccuper :

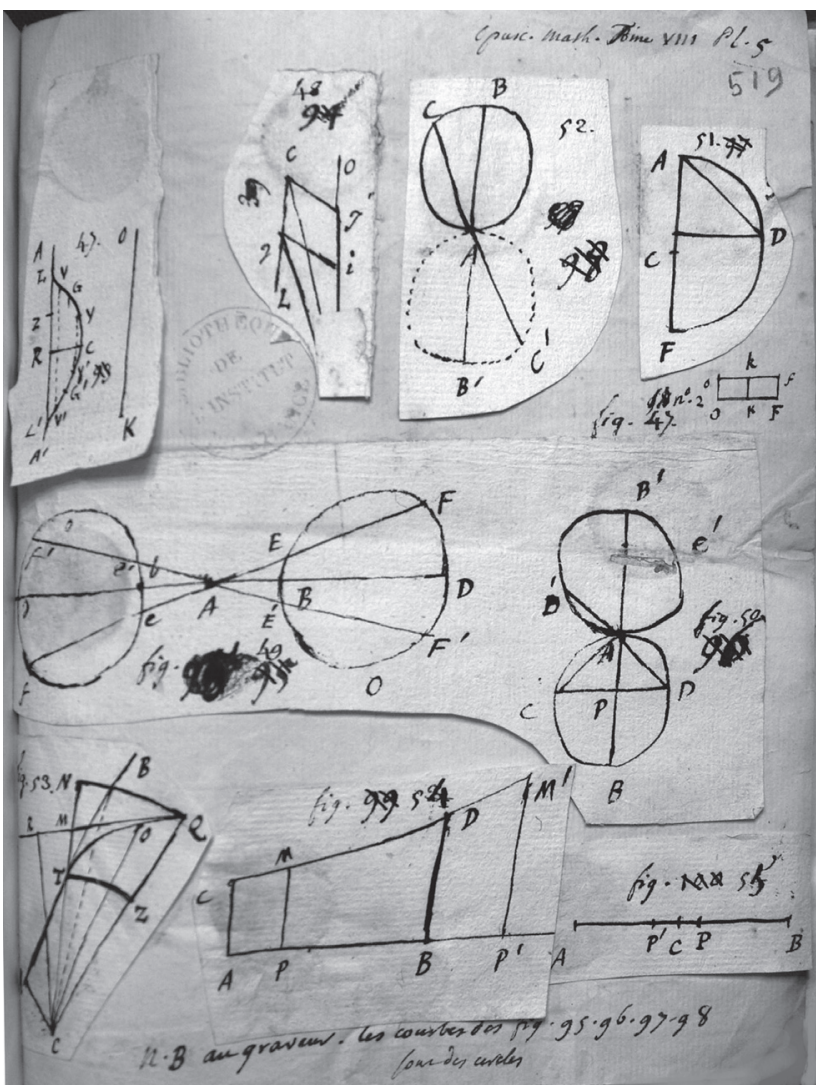

Fig. 2 : D'Alembert, Opuscules, t. VIII, planche de figures autographe no 5 avec numéros de figures biffés et remplacés (Institut de France, ms. 1788, f. 519)

En 1752, j'ai donné le premier < dans mon essay sur la résistance des fluides $>$ les vraies équations analytiques sur lesquelles est fondée la théorie dut de leur mouvement des fluides, équations vainement cherchées jusqu'alors < (j'avois déja jetté quelques germes de cette théorie dès 1746 dans mes Recherches sur la cause générale des vents) > ; Depuis ce temps là, j'ai ajouté à ces premieres recherches beaucoup d'autres recherches nouvelles et importantes sur cette théorie, 


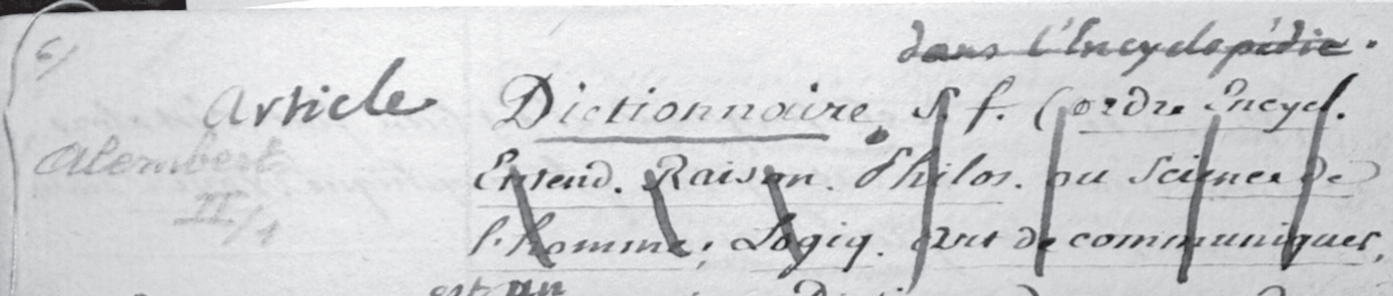

Un Jichomeive est

lequel Len wrokn a ícue longue sour distai-

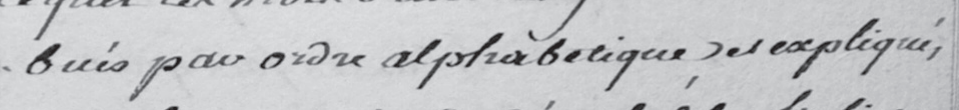
aver plun ou suoiunde détail, Selon f'objec qu'ou diepropose?

Orpeat Dinringued Groin fouler Do) Dielionsuaven, Diefioumairen de beagan, Lanque, Diefiounciren historiquen, el Diefioumaire, de Sciencenec J'areno, Jivision quion pownorit présencer Soun usupointse vue plungenerat enccle sode : Dietiouncienes de rnots, Jiefionnaives de pailh, er Diestion-

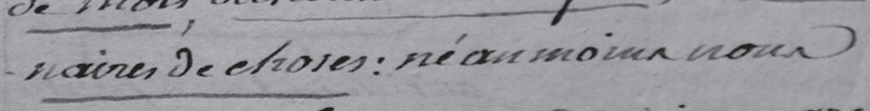
resicudzona Car premiere Division, poree quelle suan poroil pluen commode ees meince ples précife?

Eneffer un Dietionmaire) de alauguefl, quiparsoit n'ére quium Diefionnsione? De enoh, Doirêre Couvens un Dieliounciore.

Fig. 3 : D'Alembert, Dictionnaire, copie de l'article de l'Encyclopédie avec corrections autographes (coll. particulière)

(72) 
\& de celebres Geometres, entr'autres Mrs de la Grange \& Euler, y ont joint aussi les leurs, deduites avec beaucoup de sagacité et de savoir des principes que j'avois donnés (ms. 1793, f. 553).

En cette année 1752, D’Alembert était alors principalement occupé par la rédaction d'un autre pan de son œuvre scientifique : les articles mathématiques et physicomathématiques de l'Encyclopédie ${ }^{13}$.

\section{Le corpus encyclopédique}

Tous les historiens travaillant sur l'Encyclopédie ont rêvé de retrouver les manuscrits de la grande entreprise des Lumières, que ce soit pour en comprendre la fabrication, en termes de construction à partir de ses matériaux composites, traductions, compilations, créations, ou la reconstruction en termes de travail éditorial et de censure. Quel regard les deux éditeurs, Diderot et D'Alembert, exerçaient-ils sur les articles de leurs collaborateurs ? Comment les sources matérielles (traductions, manuscrits de Formey, ouvrages de référence) étaient-elles utilisées ? $\mathrm{Y}$ avait-il une nomenclature d'entrées tenue à jour au fur et à mesure des renvois ? Les manuscrits de D'Alembert n'offrent aucune réponse à ces questions puisqu'ils portent essentiellement sur la période postérieure à 1770 , et que D'Alembert a cessé son travail d'éditeur en 1758. En revanche, les quelques articles de l'Encyclopédie qui subsistent dans les papiers de D'Alembert contribuent à éclairer sa stratégie d'auteur. Nous ne traiterons ici que de ce cas emblématique et ne parlerons pas des nombreux autres autographes de D'Alembert.

Il s'agit d'une dizaine d'articles, copiés proprement, avec une marge, par un secrétaire, et portant des corrections autographes qui les font très clairement passer du statut d'articles de dictionnaire à celui d'éléments de l'œuvre d'un auteur (fig. 3). D'Alembert efface les traits caractéristiques du « genre » du dictionnaire : la typologie, aussi bien grammaticale (« substantif masculin ») qu'encyclopédique, c'est-à-dire le désignant « (Ordre Encycl. Entend. Raison. Philos. ou Science de l'homme ; Logiq. Art de communiquer, Grammaire, Dictionn.) », qui, par une chaîne ou par un simple terme, situe le mot dans l'arbre de la connaissance, et les renvois. Ces derniers, qui permettaient la circulation entre les entrées, sont soit simplement éliminés, soit remplacés par une référence explicite aux œuvres de D'Alembert publiées dans les tomes successifs de ses Mélanges : par exemple, « Voyez GRAMMAIRE » est remplacé par « Voyez dans nos Elemens de Philosophie et dans les supplémens, l'article de la Grammaire14 ».

De tels renvois, indiqués sous forme de corrections autographes en marge, fournissent déjà un premier élément de datation : les corrections de D'Alembert sont postérieures aux Mélanges d' histoire, de philosophie et de littérature, et en particulier au tome V (1767). Lorsque D'Alembert les effectue, à une ou des dates que nous ignorons, mais sur lesquelles nous allons formuler quelques hypothèses, il est manifestement motivé par une réédition de ces articles et non par une réelle mise à jour de leur contenu. Presque aucune actualisation n'intervient, en dehors des corrections de changement de statut, et de quelques améliorations de style.

Nous connaissons l'existence de quatorze15 articles autographes, copies corrigées de la main de D'Alembert, et, pour les six que nous avons pu examiner, présentés et préparés de façon similaire : Aveugle, Chronologie, Collège, Dictionnaire, Calcul difFÉrentiel, EcClésiastique, Érudition, Forme substancielle, Formulaire, Fortuit, Futur CONTINGEnt, GÉOMÈTre, GÉOMÉTRIE, GRAVITÉ16, tous antérieurs à l'interdiction de 1759. Ces articles sont-ils particulièrement importants

13. Encyclopédie, ou Dictionnaire raisonné des sciences, des arts et des métiers, par une société de gens de lettres, Paris, Briasson, David, Le Breton, Durand, 1751-1765, 17 vol. de texte.

14. Manuscrit de l'article Dictionnaire, copie avec corrections autographes, coll. privée, f. $1 \mathrm{v}^{\circ}$.

15. Quatorze, c'est bien peu sur les quelque mille sept cents articles signés $\mathrm{du}(O)$ de D'Alembert, qui ne forment eux-mêmes qu'un quarantième de l'Encyclopédie.

16. Calcul différentiel, Formulaire, Gravité sont dans le fonds Rothschild de la BnF, pièces 216 et 217 ; DictionNAIRE est maintenant dans une collection privée, après être passé en vente avec CoLlÈGE ; ÉRUDITION était entré dans les collections de la bibliothèque d'Arras, ms. 1181, détruit en 1915; GÉOMÉTRIE est dans la Eugene Smith Historical Collection (New York, Columbia) ; Aveugle, Chronologie, Ecclésiastique, Forme substancielle, Fortuit, Géomètre ne sont connus que par les catalogues de vente ; seul FUTUR CONTINGENT est à l'Institut (ms. 2472). 
pour D'Alembert? Sans aucun doute, pour lui comme pour ses contemporains : CollÈge et DictionnaIRE ont marqué les esprits. Ce sont des articles dont D'Alembert est proprement l'auteur, sans qu'une traduction ou une autre source vienne (à notre connaissance) interférer. Il est d'ailleurs intéressant de rapprocher cette liste de celle donnée par D'Alembert dans un « Mémoire sur luimême » :

Il a revu toute la partie de mathématique et de Physique generale de l'Encyclopedie, et il a meme refait en entier ou presque en entier plusieurs articles considerables \& qui contiennent des choses nouvelles, qu'on chercheroit inutilement ailleurs ; on peut citer les articles, cas irreductible, courbe, Equation, differentiel, figure de la terre, Geometrie, Infini, \&c. et un grand nombre d'autres. Outre ces articles, Mr. d'alembert en a donné à l'Encyclopedie un grand nombre de pure littérature ou de philosophie, on peut citer les articles Elemens des sciences, Erudition, dictionnaire, et plusieurs autres moins considerables, sans compter plusieurs synonymes ${ }^{17}$.

On remarque immédiatement que si D'Alembert accorde une grande importance à certains articles mathématiques, on trouve peu de science dans les articles qui nous sont parvenus. Plus précisément, on pourrait être étonné de ne pas trouver Figure DE LA TERRE, long article original, technique et méthodologique, mêlant philosophie naturelle, mathématique et géodésie, et dont D'Alembert était suffisamment fier pour en conseiller la lecture à Voltaire en 175818. Dans la même lettre, il mettait déjà en avant cinq des articles de notre liste (en italique ci-dessous) :

Je suis infiniment flatté, mon très cher et très illustre philosophe, du suffrage que vous accordez à l'article Géométrie. J'en ai fait beaucoup d'autres pour ce 7e volume dont je désirerois fort que vous fussiez content, \& où j'ai tâché de mettre de l'instruction sans verbiage, tels que force, fondamental, gravitation, gravité, forme substantielle, fortuit, fornication, formulaire, futur contingent, frères de la charité, fortune, \&c.

La liste des treize articles hors Institut, reconstituée à partir de différentes sources, figurait déjà sur un document manuscrit du XIXe siècle, une notice sur D'Alembert qui mentionnait également un certain nombre d'autres textes restés inédits 19 .

Ces indices permettent de penser que la liste d'articles de l'Encyclopédie n'est pas due au hasard de l'histoire, mais qu'elle a été délibérément construite par D'Alembert, éliminant ce qui était trop mathématique, procédé que reflètent d'ailleurs les corrections apportées à l'article Calcul difFerentiel. Les trois quarts de l'article, contenant des équations, n'ont pas été copiés et sont remplacés par un renvoi : "Voyez sur cet objet mes Mélanges de littérature et de philosophie, Tome V, $\S$ XIV, p. 239 et suiv. ou toute cette doctrine est clairement expliquée 20 ». Une note n'en est pas moins ajoutée en tête : « cet article ne peut être entendu que par les lecteurs geometres ». Nous voilà donc fixés : D'Alembert travaille pour des lecteurs, mais quels lecteurs?

La reprise d'articles de la première Encyclopédie pourrait orienter l'interprétation vers un travail de réécriture destiné à des avatars, Supplément ou Encyclopédie méthodique pour lesquels D'Alembert a été sollicité. Mais il ne s'agit ici ni de supplément, ni d'ajout, ni de mise à jour. En revanche, ce type de préparation peut être rapproché des corrections autographes portées sur certaines lettres conservées dans ses papiers, dont on peut se demander pourquoi D'Alembert les a gardées, au regard de la sélection draconienne qu'il semble avoir faite. Ainsi de la lettre d'un certain Saboureux de Fontenay 21 dont le manuscrit original présente des corrections de D'Alembert, qui la destine à l'impression :

\begin{abstract}
L A Monsieur D'Alembert-xxx
*Cette lettre m'a eté ecrite $\mathrm{L}$ par un sourd et muet de naissance \# note \# cette lettre est fidelement imprimée sur l'original, ecrit de la propre main du sourd et muet, d'un caractere très net, et d'une orthographe très exacte. Quoiqu'elle ne contienne rien d'interessant la singularité l'etat de celui qui l'a écrite semble permettre d'y faire quelque attention.
\end{abstract}

À la fin de la lettre, le nom de Saboureux et son adresse sont également biffés.

L'intérêt de D'Alembert pour les observations sur la Machine de Marly, faites par ce sourd et muet de naissance, peut être mis en relation avec l'article AvEUGLE de notre liste

17. BnF, nafr. 15551, f. 1-14, publié dans Recherches sur Diderot et sur l'Encyclopédie, $\mathrm{n}^{\circ} 38,2005$, p. 17-31.

18. O.C., vol. V/1 : lettre 58.11, 28 janvier 1758.

19. Bibliothèque historique de la Ville de Paris, Ms. 3019, f. 217.

20. BnF, nafr. 27262, pièce 216, f. 63-69.

21. O.C., vol. V/1 : lettre 76.82, [fin 1776], Institut, Ms. 2466, f. 202. 
et les préoccupations communes à Diderot et D'Alembert quant aux rapports des sens et de l'entendement. Presque trente ans séparent la Lettre sur les aveugles à l'usage de ceux qui voient (1749) de la lettre de Saboureux, mais une publication aurait pu opportunément les mettre en regard. Que D'Alembert ait eu ou non l'intention d'effectuer une publication regroupant ces textes, de nombreux documents portent les marques de telles préparations, qui se sont probablement échelonnées dans la seconde moitié des années 1770.

On peut également rapprocher de cette collection de textes le « Mémoire sur lui-même » cité plus haut, suivi, toujours de la main de D'Alembert, d'un «Supplément au mémoire précédent. Faits particuliers \& peu importans, dont on pourra faire quelque usage si on le juge à $\operatorname{propos}^{22}$ ». D'Alembert allait même jusqu'à annoncer, dans ce texte qu'il qualifie de «précis historique », une « notice » de ses ouvrages mathématiques, non retrouvée. Le contenu du mémoire permet d'en dater approximativement la rédaction à l'année 1771 .

Plusieurs indices convergents suggèrent que D'Alembert envisageait de faire une nouvelle édition de ses Mélanges, voire de ses œuvres : l'activité fiévreuse d'un «pauvre diable », Lesenne, qui se pose en intermédiaire entre l'encyclopédiste et la Société typographique de Neuchâtel (STN), et deux pages biffées contenues dans le ms. 1787 de l'Institut. Robert Darnton a parfaitement retracé les aléas et les contours mouvants du projet éditorial que la STN propose à D'Alembert au début de l'année 1780 et dont Lesenne va chercher à tirer profit, avec l'aide manifeste de D'Alembert. S'il ne fallait garder qu'un témoignage, ce serait celui d'un membre de la STN venu prospecter à Paris en mai 1780 : D'Alembert «m'a beaucoup parlé ensuite de ses ouvrages, dont il veut nous donner l'impression non point la collection entière, parce qu'il y a privilège, mais pour du neuf. Si ce n'est cette année, ce sera l'année prochaine 23 ». C'est in fine le manque d'enthousiasme de la STN devant la gloire déclinante du savant et les malheurs du pauvre diable qui enterreront ce projet. Il est clair que, de son côté, D'Alembert s'est investi dans ce projet de rassembler et préparer quelques-uns de ces "volumes de philosophie et de littérature prêts à être imprimés 24 »dont parle Condorcet en 1783. Il a ainsi pris l'initiative en 1782 de demander aux Bruyset, père et fils, de reprendre le privilège de ses Mélanges, ce qu'ils lui ont accordé pour l'" Essai de traduction de quelques morceaux de Tacite $25 »$.

C'est donc dans ce contexte d'élaboration d'œuvres complètes, ou au moins de relecture de son œuvre, entre la rédaction du «Mémoire sur lui-même » en 1771 et les projets de Lesenne en 1780, que l'on peut placer la rédaction par D'Alembert d'une réorganisation de l'ordre des textes de ses Mélanges, rassemblant les premiers éloges d'une part, les éléments de philosophie et les éclaircissements d'autre part ${ }^{26}$. Ces feuillets biffés ne seraient qu'un plan d'étape, réagencement de l'ordre de publication des textes des cinq volumes de Mélanges de 1767. Les quatorze articles de l'Encyclopédie précédemment évoqués, dont D'Alembert revendiquait la propriété intellectuelle dans son «Mémoire », et dont les manuscrits portent des marques de préparation pour une édition jamais réalisée, auraient ainsi pu constituer l'embryon d'un volume supplémentaire de Mélanges consacré à l'Encyclopédie, ultime revendication d'auteur d'une part de l'ouvrage collectif.

\section{Le corpus des éloges académiques}

Liée à la fonction de secrétaire perpétuel qu'exerce D'Alembert après 1772, une part importante des manuscrits conservés à l'Institut concerne ses activités à l'Académie française : les «Éloges » rassemblés de façon posthume dans une monumentale Histoire des membres de l' Académie française $^{27}$ (Histoire de l'Académie), proviennent pour partie d' "éloges lus » et publiés par D'Alembert en 177928 , puis repris à l'identique pour constituer le tome I

22. BnF, nafr. 15551 , f. 7.

23. R. Darnton, « The life of a "poor devil" in the Republic of letters », Essays on the age of enlightenment in honor of Ira O. Wade, éd. Jean Macary, Genève-Paris, Droz, 1977, p. 39-92.

24. Euvres de Frédéric le Grand, Preuss, t. XXV, Berlin, Decker, 1854, p. 370. 25. Institut, ms. 1787, f. 18-23.

26. Institut, ms. 1787, f. 89 et $91 \mathrm{v}^{\circ}$. Le manuscrit est biffé et le verso a été utilisé pour des calculs.

27. 6 vol. in-12, Paris, Moutard, 1785-1787.

28. Éloges lus dans les séances publiques de l'Académie française, Paris, Panckoucke, 1779 (désormais Éloges lus). 
de l'Histoire de l'Académie 29 . Afin de préciser cette situation éditoriale quelque peu complexe, l'examen des manuscrits (ms. 2740, 2472 et 2473, principalement) fournit des éléments d'appréciation, de même qu'il apporte des éclairages sur les stratégies d'écriture et de publication de D'Alembert. Ces éloges illustrent ainsi une part importante d'une pratique littéraire académique, que permet d'appréhender la présentation de ce que l'on pourrait appeler ses «matériaux », dans la mesure où ils apparaissent comme des éléments de fabrication du texte, au demeurant non seulement lacunaires mais aussi hétérogènes.

Certains documents manuscrits sont extérieurs au corpus des éloges entendu au sens strict, puisqu'ils n'émanent ni de D'Alembert, ni de l'un de ses secrétaires. Tenu, en vertu du genre même de l' «éloge académique », de détailler «toute la vie d'un académicien, depuis sa naissance jusqu'à sa mort », ainsi qu'il le rappelle dans l'article ÉLoge de 1'Encyclopédie 30, D'Alembert doit faire face à un déficit d'informations concernant certains personnages : il fait paraître dans les journaux des appels à témoignage 31 et entre parfois directement en contact avec les descendants de certains académiciens. Pour preuve, un ensemble de pièces relatives à Jacques Adam, secrétaire des commandements du prince de Conti (16631735), envoyé par son fils Louis-Henri : une lettre, non datée, invite D'Alembert à enrichir l'« essai » qu'il lui a communiqué « des circonstances » qu'il voudra bien se « donner la peine d'extraire » de son « exposé », lequel consiste en deux récits retraçant la vie de l'académicien, le premier précédé d'un titre, ajouté de la main de D'Alembert, « Sur Mr. Adam, par son fils ${ }^{32}$ ». La confrontation entre ces pièces et la version imprimée de l'article sur Jacques Adam ${ }^{33}$ révèle ainsi la manière dont D'Alembert s'approprie ces éléments qui deviennent par là partie intégrante de la fabrique de son texte, fondée sur une pratique du réemploi et de la réécriture.

Mais le corpus manuscrit comporte surtout d'autres éléments internes au processus d'élaboration des textes, qui bénéficient, à des degrés divers, du statut d'avant-textes. C'est le cas, par exemple, à propos de l'éloge d'Esprit Fléchier (1632-1710), évêque de Nîmes, d'un ensemble de six feuillets comportant des notes préparatoires ${ }^{34}$ (fig. 4). Ce document, constitué d'une suite de courts développements, plus ou moins rédigés, séparés par des traits horizontaux, présente un caractère programmatique évident : il s'agit d'une liste de points à aborder dans le texte, dans un ordre qui n'est pas celui du texte imprimé35, ce qui confère au document moins le statut de plan que celui de réservoir de données, dans une phase précoce du processus d'invention. C'est ce que suggère l'ajout de numéros, modifiables par la suite, qui seraient la trace du travail de disposition. C'est aussi ce que suggère le fait que tous les développements ont été biffés, la biffure témoignant de l'accomplissement, ailleurs et ultérieurement, de la rédaction de chaque développement présent ici à l'état embryonnaire : confrontant son texte avec les éléments contenus dans le document, D’Alembert vérifierait en somme qu'il n'a rien oublié de son programme initial. Ce retour sur les éléments constitutifs de l'éloge se lit aussi dans les corrections apportées aux bribes de texte, soit contemporaines de la rédaction de ce brouillon, soit de la phase de relecture et de vérification. Cette dernière hypothèse est d'autant plus probable que la confrontation du document avec d'autres matériaux conservés montre que certains éléments du brouillon ont été rajoutés dans un état plus tardif du texte 36 .

D'autres avant-textes de l'éloge de Fléchier révèlent en effet la complexité du processus de rédaction du texte de l'éloge et des notes qui s'y rapportent, ainsi que les difficultés rencontrées dans l'exploitation des manuscrits conservés, en particulier pour l'étude génétique.

29. Dans les Éloges lus, D'Alembert ne publie pas les notes qui se rapportent aux textes des éloges, qui paraîtront dans les tomes II à VI de l'Histoire de l'Académie. Dans ces cinq derniers tomes, les notes sont systématiquement imprimées à la suite du texte de l'éloge.

30. Encyclopédie, éd. cit., t. V (1755), p. 527 b.

31. Voir, par exemple, l'avis du 18 janvier 1774 publié dans le Journal encyclopédique, février 1774, p. 519-521.

32. Institut, ms. 2472 , f. $95 \mathrm{r}^{\circ}-\mathrm{v}^{\circ}$, f. $90 \mathrm{r}^{\circ}-94 \mathrm{r}^{\circ}$, f. $96 \mathrm{r}^{\circ}-102 \mathrm{r}^{\circ}$, respectivement.

33. Histoire de l'Académie, éd. cit., t. IV, p. 571-585.

34. Institut, ms. 2473 , f. $450 \mathrm{r}^{\circ}$-f. $455 \mathrm{v}^{\circ}$.

35. Histoire de l'Académie, éd. cit., t. I, p. 387-429 [texte de l'éloge] ; t. II, p. 391-437 [notes].

36. Les analyses consacrées à l'éloge de Fléchier exploitent l'étude du manuscrit effectuée par Vincent Barrellon, élève-ingénieur à l'École centrale de Lyon. 


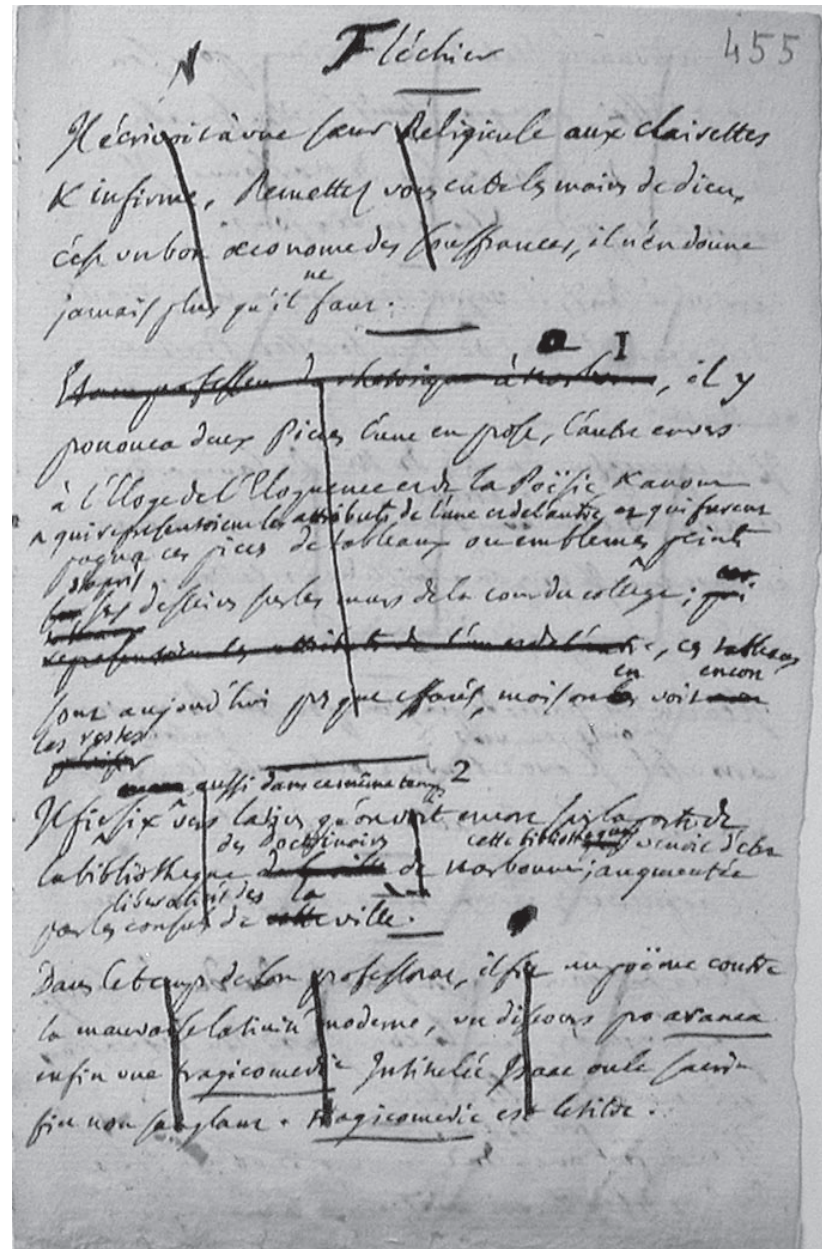

Fig. 4 : D’Alembert, Début des notes préparatoires autographes pour la rédaction de l'Éloge de Fléchier (Institut de France, ms. 2473, f. 455 r $^{\circ}$ )

Contrairement à d'autres éloges pour lesquels il existe une ou plusieurs versions complètes, les matériaux sont ici lacunaires et impliquent un travail de reconstitution, fondé sur des hypothèses prenant en compte et combinant plusieurs ordres de considérations.

La comparaison avec l'imprimé des fragments de textes conservés convainc rapidement qu'il s'agit d'éléments discontinus, relatifs aux deux grands ensembles que constituent le texte de l'éloge, d'une part, les notes sur le texte et le «Supplément » qui les prolonge, d'autre part. Ce premier regroupement rudimentaire en suppose cependant un second, en raison de l'extrême éparpillement des feuillets, dispersés dans les trois recueils manuscrits signalés plus haut ${ }^{37}$. Plusieurs démarches permettent d'entreprendre un tel travail, qui montre que l'on a affaire à un ensemble non seulement discontinu, mais aussi dont les fragments constitutifs correspondent à des phases de rédaction différentes de l'éloge.

Une démarche consiste à se fonder sur des indices matériels. Les uns concernent la main du scripteur : on distingue aisément les mains de deux secrétaires différents, ce qui invite à apparier les fragments copiés par l'un et l'autre 38 ; on reconnaît aussi la main de D'Alembert, qui corrige les textes copiés et rédige certains fragments. Les autres concernent des marques par lesquelles D'Alembert matérialise la jonction entre des passages disposés sur des feuillets différents : reprise d'une séquence de termes ; signes diacritiques (dièses, croix cerclées, etc.).

Une autre approche consiste à effectuer la confrontation des textes en identifiant en particulier les phénomènes de reprise : les corrections autographes indiquées sur tel passage sont, sur un autre feuillet, prises en compte dans la copie, nécessairement ultérieure, du même passage. La combinaison de ces deux démarches, en lien avec l'examen du texte de l'imprimé, considéré comme un point d'aboutissement, conduit à l'amorce d'une chronologie relative des fragments. Cette chronologie demeure toutefois imparfaite : certains fragments, qui ne présentent pas de portions textuelles permettant une comparaison, ne peuvent être mis en rapport avec aucun autre.

Le corpus manuscrit des éloges présente fort heureusement des cas plus simples, qui permettent de pousser plus avant la réflexion. Certains éloges ont une unique version manuscrite, d'autres, deux successives : la première, de la main d'un secrétaire, présente des corrections autographes qui sont intégralement reportées dans la deuxième, avant que D'Alembert n'effectue une nouvelle campagne de corrections. Pour illustrer le

37. On dispose actuellement de dix fragments différents : cinq concernent le texte, quatre les notes, un dernier le texte et les notes.

38. Critère nécessaire, mais non suffisant, dans la mesure où d'autres indices portent à penser que les secrétaires travaillent occasionnellement en parallèle sur le texte du même éloge. 


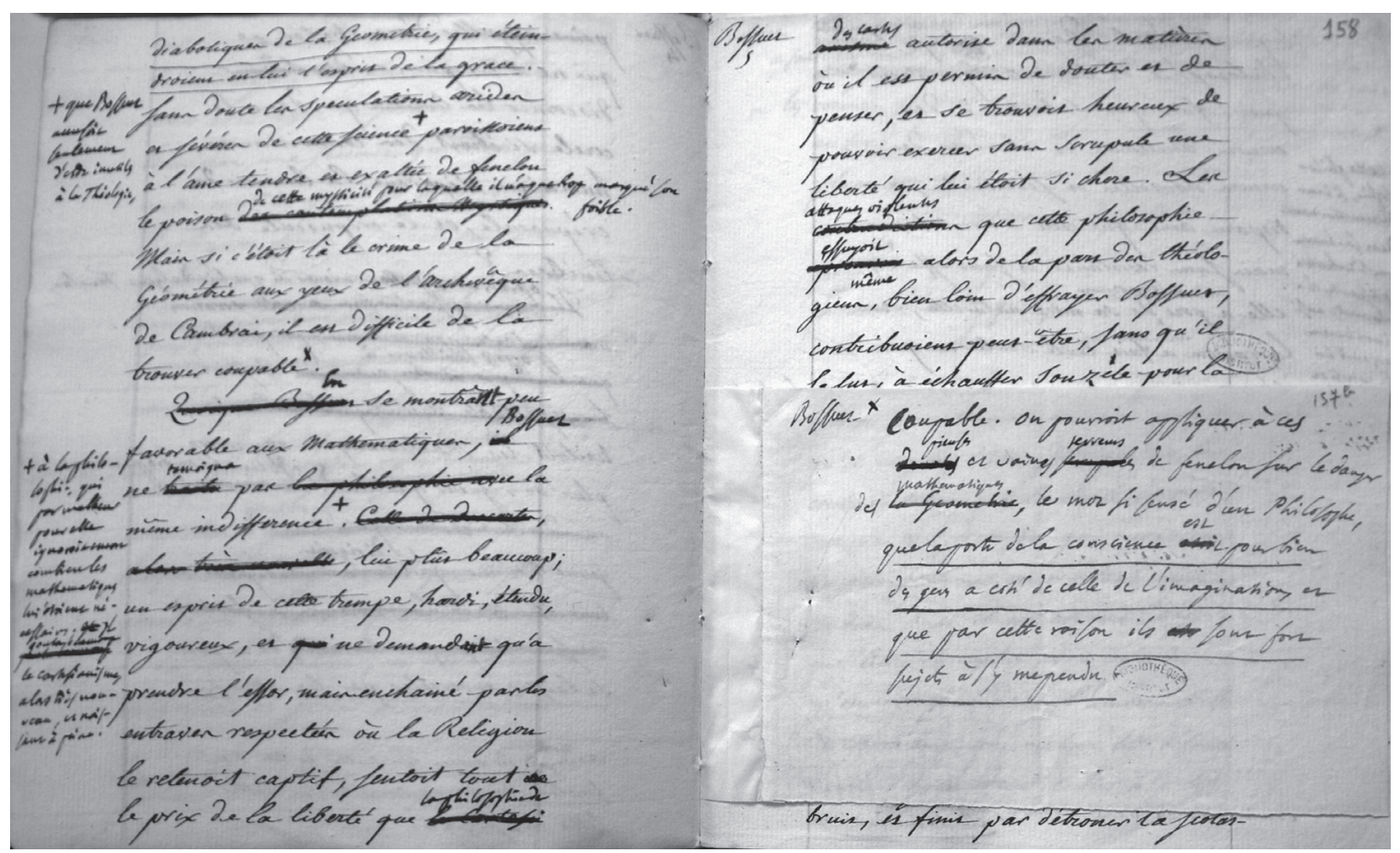

Fig. 5 : D’Alembert, Éloge de Bossuet : copie de la première version avec corrections autographes et ajout d'une paperole autographe repérée par un signe diacritique (croix) et comportant la reprise du mot («coupable ») à la suite duquel doit se placer l'ajout (Institut de France, ms. 2473, f. 157 v $^{\circ}$, f. $157 \mathrm{~b}$ (paperole) et f. 158 r $^{\circ}$ )

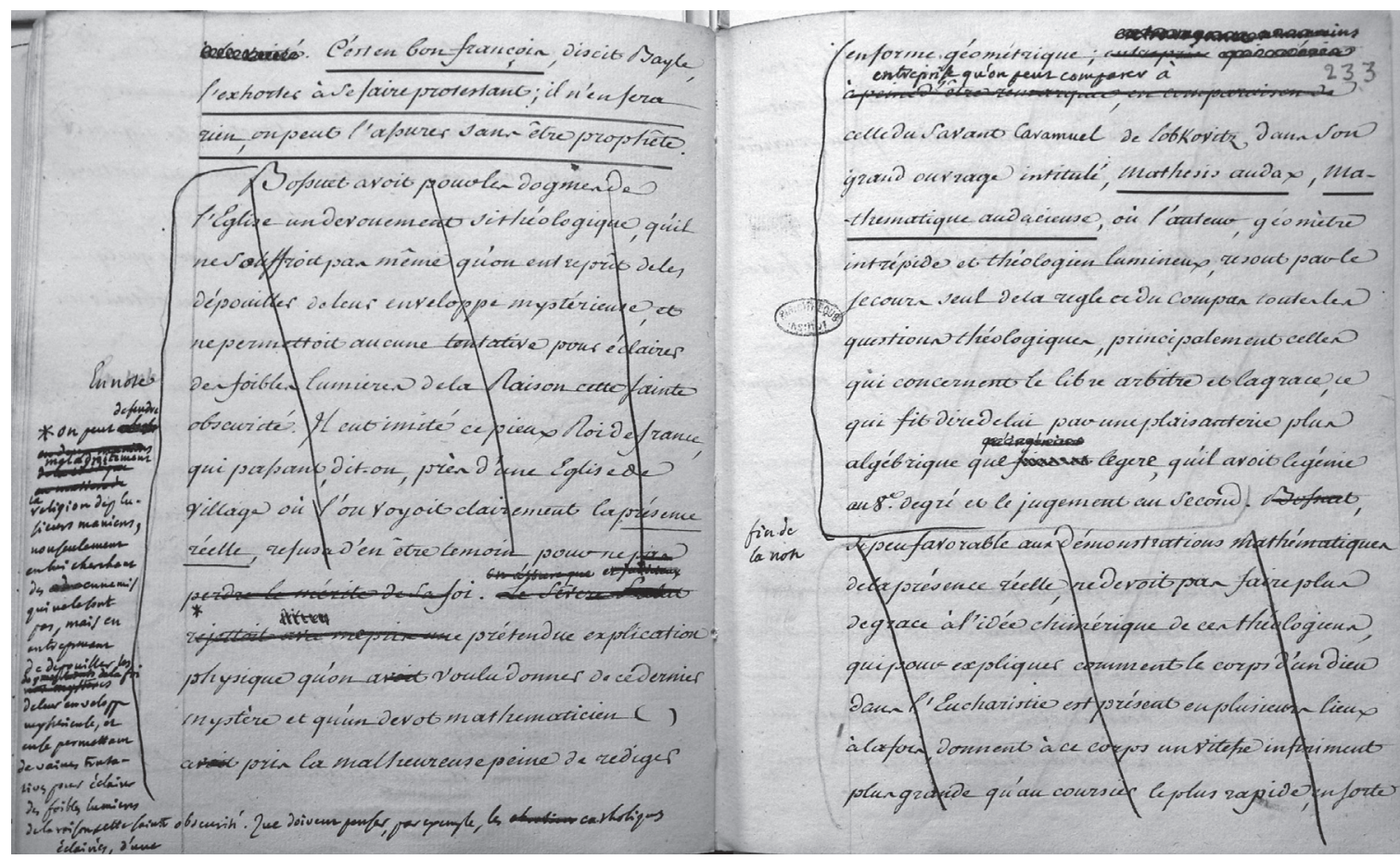

Fig. 6 : D’Alembert, Éloge de Bossuet : copie de la deuxième version avec corrections autographes et indication d'un déplacement « En note» (Institut de France, ms. 2473, f. $232 \mathrm{v}^{\circ}-233 \mathrm{r}^{\circ}$ ) 
phénomène, nous privilégierons l'éloge de Bossuet (16271704), d'une part parce qu'il est le plus long et donc susceptible de présenter à grande échelle une multitude de phénomènes récurrents, d'autre part parce qu'il s'agit d'un «éloge lu », publié en deux temps : le texte paraît d'abord en 1779 ; les notes sont publiées de manière posthume.

\begin{tabular}{|c|c|c|c|c|}
\hline \multirow{3}{*}[\ldots]{} & MS 1 & MS 2 & \multirow{3}{*}[\ldots]{} & IMPRIMÉ \\
\hline & \multirow{2}{*}{$\begin{array}{c}\text { Texte de l'éloge } \\
(\mathrm{ms} .2473, \\
\left.\text { f. } 154 \mathrm{r}^{\circ}-202 \mathrm{v}^{\circ}\right) \\
\text { [copie avec } \\
\text { corr. autogr.] }\end{array}$} & $\begin{array}{l}\text { Texte de l'éloge } \\
(\mathrm{ms} .2473, \\
\left.\text { f. } 203 \mathrm{r}^{\circ}-243 \mathrm{r}^{\circ}\right) \\
\text { [copie avec } \\
\text { corr. autogr.] }\end{array}$ & & $\begin{array}{c}\text { Éloge de } \\
\text { Bossuet } \\
\text { (Éloges lus, } \\
\text { p. 133-174) }\end{array}$ \\
\hline & & $\begin{array}{c}\text { « Notes sur } \\
\text { l'article de } \\
\text { Bossuet } \\
(\mathrm{ms} .2473, \\
\left.\text { f. } 244 \mathrm{r}^{\circ}-283 \mathrm{r}^{\circ}\right) \\
\text { [copie avec } \\
\text { corr. autogr.] }\end{array}$ & & $\begin{array}{c}\text { Notes sur } \\
\text { l'Éloge de } \\
\text { Bossuet } \\
\text { (Histoire de } \\
\text { l'Académie, } \\
\text { t. II, } \\
\text { p. 221-291) }\end{array}$ \\
\hline
\end{tabular}

Le corpus manuscrit de cet éloge n'en demeure pas moins lacunaire : outre le fait que l'on ignore quel texte a été lu par D'Alembert lors de la séance publique du 15 mai 1775 , la deuxième version manuscrite ne coïncide pas strictement avec le texte de l'imprimé, ce qui suppose l'existence d'une ou plusieurs versions manuscrites ultérieures. Reste que l'ensemble conservé fait apparaître la mise en place d'une économie textuelle dynamique que l'on peut interpréter, par l'étude de détail, en termes de stratégie d'écriture, mais aussi de stratégie éditoriale.

Outre les corrections ponctuelles que D'Alembert apporte sur chacune des versions, on remarque, de l'une à l'autre, un phénomène d'expansion qui résulte de nombreux ajouts, dans l'interligne, dans les marges voire, pour les portions qui, par leur ampleur, nécessitent le recours à un autre support matériel, sur ce que l'on désignera, à la suite de Proust, comme des « paperoles » (fig. 5). Nombre de ces ajouts concernent les « réflexions philosophiques » que D'Alembert affirme devoir former, dans les éloges, « comme des masses de lumiere qui serviront à éclairer le reste » : des réflexions inspirées par l'« esprit philosophique » dont il se réclame aussi dans la «Préface »des Éloges lus ${ }^{39}$. On observe aussi, dans le deuxième manuscrit, l'apparition d'une section intitulée « Notes sur l'article de Bossuet », qui constitue le noyau de ce qui devient, dans l'imprimé, l'ensemble textuel des notes qui se rapportent au texte de l'éloge.

Or, au processus d'expansion du texte se combine un phénomène de déplacement, marqué par la présence de notes de régie à l'intention du secrétaire ${ }^{40}$, précisant que tel passage, isolé par des traits, doit être déplacé « en note »- ce qui n'exclut par ailleurs pas la présence de corrections supplémentaires, apportées avant ou après le choix d'effectuer ce déplacement (fig. 6). Le phénomène attire ainsi l'attention sur l'importance des notes, dont l'existence est attestée dès les premières versions de l'éloge : de courtes notes, disposées en bas de page, figurent dans la première version manuscrite connue ; dans l' «Avertissement sur les éloges qui suivent », publié dans le recueil de 1779 , D'Alembert faisait déjà état de «beaucoup de notes » rédigées, qu'il lui a «paru à propos de supprimer quant à présent »(Éloges lus, p. II).

L'examen des remaniements effectués par D'Alembert révèle l'existence d'une économie textuelle particulière qui est d'abord, au sens strict, une économie d'écriture. Les déplacements effectués illustrent un mode de composition fondé sur la pratique généralisée du réemploi : rien ne se perd, ou presque, dans le texte, la plupart des passages coupés ici se retrouvant collés là, en particulier dans les notes, dont l'élaboration s'apparente à un travail proche de la marqueterie. Mais la démarche va sans doute au-delà du seul souci rhétorique d'équilibrer des masses textuelles: le déplacement en note de certains passages paraît aussi orienté par une stratégie éditoriale.

D'une part, l'étude du détail des corrections autographes fait apparaître une accentuation significative de la charge critique à l'encontre de Bossuet, qui s'explique, sans surprise, par les divergences idéologiques entre le «philosophe » D'Alembert et l'un des représentants les plus éminents de l'orthodoxie catholique. Ce positionnement critique s'exprime en particulier à propos des querelles

39. Encyclopédie, t. V, p. 527 b ; Éloges lus, éd. cit., p. XIX. 40. Phénomène impliquant l'existence d'au moins une version ultérieure, non retrouvée. 
religieuses et du rôle joué par l'évêque de Meaux dans la mise en œuvre de la politique d'intolérance, notamment à l'égard des « hérétiques »- entendons, des protestants -, consécutive à la Révocation de l'édit de Nantes (1685). D'autre part, force est de constater que ce sont souvent les passages les plus ouvertement militants qui, au terme des processus de déplacement signalés, se retrouvent « en note », c'est-à-dire précisément dans la portion du texte qui, en 1779, demeure « quant à présent » à l'état manuscrit.

On peut alors faire l'hypothèse que cette convergence des remaniements est sous-tendue par une stratégie de publication différée ${ }^{41}$, qui a partie liée avec la dialectique qu'affrontent les «philosophes » des Lumières et, avant eux, nombre de "libres penseurs », de ce qui peut être écrit et de ce qui peut être publié. Il ne faut pas sous-estimer l'importance du statut d'une production soumise à approbation et privilège, qui, signée par le secrétaire perpétuel de l'Académie française, engage aussi l'institution. On ne doit pas non plus négliger ce que Condorcet désigne, dans l'« Épître dédicatoire » qui ouvre le tome II de l'Histoire de l'Académie, comme une aspiration au « repos » (p. II), sans doute légitime après l'expérience violente qu'a représentée la querelle de l'Encyclopédie.

Cette stratégie éditoriale s'avère néanmoins ambivalente, et l'existence, dans le corpus manuscrit, de fragments demeurés inédits soulève la question du vieillissement du discours : en particulier, les lecteurs des notes imprimées sur l'éloge de Bossuet ne connaîtront pas deux développements exposant des « reflexions sur les traverses que le progres des lumieres a essuyées dans tous les siècles » et d'autres « reflexions » en faveur de l'instauration d'une tolérance ecclésiastique et civile 42 qui, à l'initiative de Condorcet ou, plus probablement, de D'Alembert, ne paraissent pas dans l'Histoire de l'Académie. Si cette disparition s'explique, les années passant, par le caractère de moins en moins pertinent de ce qui pouvait apparaître, au moment de la rédaction, comme un discours militant, la nature de ces fragments manuscrits relance l'interrogation sur le statut des notes et sur les relations qu'elles entretiennent avec le texte de l'éloge : ces deux passages, d'autant plus faciles à supprimer qu'ils se présentent comme des digressions, prennent la forme de morceaux détachables, qui ont une physionomie proche de celle de certains fragments que Voltaire publie dans ses volumes de mélanges. Est ici en question la relative indépendance des notes par rapport au texte, ce qui n'est pas sans incidence sur la poétique des éloges « philosophiques » que théorise D’Alembert.

$\mathrm{Si}$ les caractéristiques principales de l'économie textuelle de l'éloge de Bossuet se retrouvent dans quelques autres éloges du corpus manuscrit, ces cas demeurent néanmoins faiblement représentés, peut-être en raison même des aléas de l'histoire de sa conservation. L'analyse gagnera à être affinée par l'exploration d'un tel corpus, jusque-là resté méconnu.

Le corpus des « manuscrits de D'Alembert » s'avère en effet déconcertant à bien des égards. La dimension fragmentaire, tardive et souvent dispersée de l'ensemble résulte en partie de son histoire complexe. Face à tel manuscrit, il est souvent difficile de savoir non seulement à quelle phase de rédaction du texte on a affaire, mais aussi pour quelles raisons cette version a été conservée plutôt que d'autres. La question s'entend même plus généralement, si l'on considère le caractère également composite d'un corpus qui comporte des autographes de D'Alembert et des copies, avec ou sans corrections autographes. La part, significativement prépondérante, des manuscrits portant la trace de la main de D'Alembert pourrait suggérer une première explication : les manuscrits ont peut-être d'abord été conservés parce qu'il s'agit de manuscrits d'auteur, qui présenteraient à ce titre une plus grande valeur que d'autres, rédigés par des mains étrangères, peut-être même aussi que des copies autographes de passages d'ouvrages susceptibles de servir de sources qui ont sans doute existé. Reste, entière, la question de l'origine de la sélection : est-ce D'Alembert lui-même qui a effectué un tri dans les papiers qu'il a légués à Condorcet ou son légataire et ses héritiers en ont-ils pris l'initiative ? Toujours est-il que, face à l'alternative entre matérialité et auctorialité, la logique de l'auteur semble dans une certaine mesure avoir prévalu.

41. Voir O. Ferret, «Les "réflexions philosophiques" dans les éloges académiques de D'Alembert : le cas de l'Éloge de Bossuet », D'Alembert i Lumi, l'Europa/D'Alembert, les Lumières et l'Europe, éd. P. Crépel et Luigi Pepe, Bollettino di storia delle scienze matematiche, vol. XXVIII, 2008 , fasc. 2, p. 255-272.

42. Institut, ms. 2473 , f. $246 \mathrm{r}^{\circ}-252 \mathrm{v}^{\circ}$; f. $259 \mathrm{v}^{\circ}-270 \mathrm{v}^{\circ}$. 
C'est aussice que confirmeraient les stratégies éditoriales que nous avons tenté de mettre au jour, qui font néanmoins apparaître deux principaux cas de figure. Celui, d'une part, des nombreux manuscrits que D'Alembert n'entend ou ne peut pas publier, du moins « quant à présent », révélant ainsi une stratégie de publication différée. Si elle peut s'expliquer, en ce qui concerne l'Histoire de l'Académie, par le calcul de prudence du secrétaire perpétuel de l'Académie française, d'autres textes, par exemple certains articles de l'Encyclopédie, sont manifestement préparés pour une publication séparée ou, s'agissant des nombreux mémoires académiques de la première période de son activité scientifique, conservés et retravaillés en vue d'un passage à l'imprimé. On aurait alors affaire à une stratégie d'auteur, dans sa double composante d'homme de lettres et de sciences, soucieux de «faire œuvre ». D'autre part, les derniers tomes des Opuscules, rédigés et publiés sans attendre, reflètent l'image d'un savant de renom qui peut se permettre d'écrire pour une petite poignée d'illustres confrères, sans se soucier du public de ses premières années, en particulier académique.
L'examen du corpus manuscrit, si tant est que les manuscrits fassent « corpus », permet aussi d'observer la mise en place d'une économie d'écriture qui frappe par sa constance, que les sujets abordés soient « scientifiques » ou « littéraires ». Il en va de la gestion de l'imprimé comme du manuscrit, et des manuscrits de l'auteur comme de ceux des autres : D'Alembert pratique systématiquement et à grande échelle le réemploi des textes, qui circulent et se modifient au sein de cette « œuvre » dont le périmètre est mouvant, dans ses frontières comme dans la physionomie, en incessante reconfiguration, de ses éléments constitutifs. Une telle particularité dans le mode de production des textes n'est pas sans poser un certain nombre de défis aux éditeurs des Euvres complètes : quel statut, entre source et avant-texte, accorder aux différents « papiers » ? Comment rendre compte des remaniements de tous ordres dont les manuscrits portent la trace ? En somme, prendre pour objet l'examen des « manuscrits de D'Alembert » conduit à un questionnement en prise sur des problématiques qui sont indissociablement celles de la génétique textuelle et de l'édition critique. 
G $\quad$ E $\quad \mathrm{N} \quad \mathrm{E} \quad \mathrm{S} \quad \mathrm{I} \quad \mathrm{S}$

OLIVIER FerRet enseigne la littérature française du XVIII e siècle à l'université Lyon II ; il est membre de l'Institut universitaire de France et membre statutaire de LIRE (Littérature, idéologies, représentations aux XVIII ${ }^{\mathrm{e}}$-XIXe siècles), UMR 5611 du CNRS.

Alexandre Guilbaud est historien des mathématiques à l'université Pierre et Marie Curie, membre de l'Institut de mathématiques de Jussieu, UMR 7586 du CNRS.

IrÈne Passeron est historienne des sciences, chargée de recherches au CNRS, dans l’UMR 8630, SYRTE (Systèmes de référence temps espace), Observatoire de Paris et université Pierre et Marie Curie.

Tous trois participent à l'édition des Euvres complètes de D'Alembert (Paris, CNRS Éditions).

Olivier.Ferret@univ-lyon2.fr

alexandre.guilbaud@upmc.fr

irene.passeron@obspm.fr

Résumés

Les manuscrits de D'Alembert, correspondance, notes et brouillons ou écrits inédits et projets de rééditions, soulèvent la question de leur histoire et de leur statut dans son œuvre. Ils constituent une source riche et complexe : révélatrice de sa façon de travailler mais dont l'hétérogénéité doit être évaluée. Écrits « scientifiques » et « littéraires », zones compactes et facilement identifiables aussi bien que sous-groupes de manuscrits parsemés et lacunaires paraissent souvent inclassables. Nous rendons compte de ces « pleins et déliés », de leurs promesses et de leurs exigences à partir de trois cas : les manuscrits scientifiques de D'Alembert, témoins de l'évolution de son mode de rédaction et de publication ; les copies corrigées de ses articles de l'Encyclopédie, préparatoires à une réédition organisée du même type que celle entreprise dans ses Mélanges ; les versions manuscrites, enfin, de certains de ses Éloges, témoins singuliers de son économie d'écriture.

D'Alembert's manuscripts, correspondence, notes, drafts, unpublished writings and reissue projects raise the question of their history and their status in his works. They form a rich and complex source, revealing D'Alembert's working methods, but their heterogeneity has yet to be evaluated. Scientific and literary writings, compact and easily identifiable zones as well as subsections of dispersed and incomplete manuscripts, often appear impossible to classify. We show these "downstrokes and upstrokes", their promises and requirements in three cases: D'Alembert's scientific manuscripts, evidence of the evolution of his writing and publication methods; the corrected copies of his Encyclopedia articles, preliminary to the organized reissue similar to the one undertaken in his Mélanges; and finally, the manuscript versions of some of his Éloges, uncommon evidence of his writing system.

Die Handschriften D'Alemberts, seine Korrespondenz, Notizen und Entwürfe, oder auch nicht editierten Schriften und Pläne für Neuausgaben werfen die Frage nach ihrer Geschichte und ihrem Status im Gesamtwerk auf. Sie bilden eine reiche und vielfältige Quelle für das Studium seiner Arbeitsweise, deren Heterogenität allerdings erst evaluiert werden muss. „Wissenschaftliche“ und ,literarische“ Schriften, zusammenhängende und leicht zu identifizierende Bereiche, aber auch Untergruppen heterogener und lückenhafter Manuskripte, die oft unklassifizierbar erscheinen. Wir geben Rechenschaft von all ihren Schätzen und Möglichkeiten, indem wir von drei Fällen ausgehen: die wissenschaftlichen Manuskripte D'Alemberts als Zeugen der Entwicklung seiner Schreib- und Veröffentlichungsweise; die korrigierten Abschriften seiner Encyclopédie-Einträge als Vorbereitungen für eine Neuausgabe (ähnlich wie die seiner Mélanges); und endlich die handschriftlichen Fassungen bestimmter Éloges als einzigartige Zeugen seiner Schreibstrategie.

\section{Pleins et déliés dans les manuscrits de D’Alembert}

Los manuscritos de D'Alembert, correspondencia, notas y borradores, o escritos inéditos y proyectos de reedición plantean la cuestión de su historia y de su situación con respecto a la obra, constituyendo una fuente rica y compleja, que revela su manera de trabajar, pero cuya heterogeneidad debe ser evaluada. Escritos "científicos" y "literarios", zonas compactas y fácilmente identificables junto con subgrupos de manuscritos dispersos y fragmentarios que aparecen a menudo como inclasificables. Damos cuenta aquí de esos "claroscuros", de sus promesas y sus exigencias a partir de tres casos: los manuscritos científicos de D'Alembert, testimonios de la evolución de su manera de redactar y publicar; las copias corregidas de sus artículos de la Enciclopedia, preparatorias de una reedición del mismo tipo que la experimentada en sus Mélanges, y, finalmente, las versiones manuscritas de algunos de sus Éloges, testimonios singulares de su economía de escritura.

I manoscritti di D'Alembert - lettere, note, appunti o scritti inediti, e progetti di riedizione - sollevano la questione della loro storia $\mathrm{e}$ del loro statuto nella sua opera. Costituiscono una fonte ricca e complessa, rivelatrice del suo modo di lavorare, ma la loro eterogeneità necessita una valutazione: scritti "scientifici" e "letterari", zone compatte e facilmente identificabili, ma anche sottogruppi di manoscritti sparsi e lacunosi, spesso non classificabili. Qui analizziamo queste diversità, le loro promesse e le loro esigenze, a partire da tre casi: i manoscritti scientifici di D'Alembert, testimoni dell'evoluzione del suo modo di redigere e pubblicare; le copie corrette de suoi articoli dell'Encyclopédie, preparatori a una riedizione organizzata dello stesso tipo di quella delle Mélanges; infine le versioni manoscritte di alcuni dei suoi Éloges, esempi singolari della sua economia di scrittura.

Os manuscritos de D'Alembert-correspondência, notas e rascunhos ou escritos inéditos e projectos de reedição - colocam a questão da sua história e do estatuto que ocupam na sua obra. Fonte rico e complexa, eles são reveladores do seu modo de trabalhar, mas têm uma heterogeneidade que precisa de ser avaliada. Escritos "científicos" e "literários", zonas compactas e facilmente identificáveis ao lado de conjuntos de manuscritos dispersos e lacunares, tudo isso resiste a uma classificação imediata. Damos aqui conta dessa mescla de traços cheios e finos, com as suas promessas e exigências, a partir de três casos: os manuscritos científicos de D'Alembert, testemunhos de evolução no seu modo de redacção e publicação; as cópias corrigidas dos seus artigos da Enciclopédia, preparatórios de uma reedição organizada à semelhança das Mélanges; e, por último, as versões manuscritas de alguns Éloges, testemunhos singulares da economia da sua escrita. 